\title{
OS DESAFIOS DO USO DE TRILHAS EM UNIDADES DE CONSERVAÇÃO
}

\author{
Vanessa Leite Rezende ${ }^{1}$
}

Fernanda Leite Cunha ${ }^{2}$

\begin{abstract}
RESUMO
A utilização de trilhas é um dos principais objetivos dos visitantes das Unidades de Conservação, e vem se tornando cada vez mais constante. Porém, essa procura pode causar diversos impactos a essas áreas protegida. $O$ presente trabalho visou levantar os principais pontos positivos e negativos do uso das trilhas na Unidades de Conservação do Brasil através de uma revisão da literatura existente. Foi encontrado diversos impactos causados aos fatores bióticos e abióticos, como alterações no solo, luz e temperatura, relacionados a abertura ou uso das trilhas, o que pode provocar alterações a biodiversidade local, além de prejudicar os serviços ecossistêmicos gerados por essas áreas. No entanto, o uso de ferramentas de Educação Ambiental, o planejamento da manutenção e a fiscalização por parte do gestor da Unidade de Conservação pode minimizar esses impactos e assim gerar benefícios para a população local e para os visitantes no geral, através do aprendizado de práticas conservacionistas.
\end{abstract}

PALAVRAS-CHAVE: Ecoturismo. Educação Ambiental. Uso Público de Unidades Conservação

\section{THE CHALLENGES OF THE TRACKS OF USE IN STORAGE}

\section{UNITS}

\begin{abstract}
The use of trails is one of the main objectives of the visitors of the protected areas, and is becoming more and more constant. However, this demand can cause several impacts to these protected areas. This paper examines the main strengths and weaknesses of the use of trails in Brazil's protected areas through a literature review. It was found several impacts to biotic and abiotic factors, such as changes in the soil, light and temperature, related to opening or use of trails, which can cause local biodiversity changes, in addition to affecting the ecosystem services generated by these areas. However, the use of environmental education tools, maintenance planning and review by the Conservation Unit manager can minimize these impacts and generate benefits for local people and visitors in general, through learning practices conservationists.
\end{abstract}

KEYWORDS: Ecotourism. Environmental Education. Public Conservation Units Use

\footnotetext{
${ }^{1}$ Doutoranda em Biologia Vegetal, Universidade Federal de Minas Gerais. vanessa.leite.rezende@gmail.com

${ }^{2}$ Graduanda em Engenharia Florestal, Universidade Federal de Lavras. fernandaleitecunha@gmail.com
} 


\section{LOS RETOS DE LAS PISTAS DE USO EN UNIDADES DE ALMACENAMIENTO}

\section{RESUMEN}

El uso de los senderos es uno de los principales objetivos de los visitantes de las áreas protegidas, y se está convirtiendo cada vez más constante. Sin embargo, esta demanda puede causar varios impactos en estas áreas protegidas. Este documento examina las principales fortalezas y debilidades de la utilización de senderos en las áreas protegidas de Brasil a través de una revisión de la literatura. Se encontró varios impactos a factores bióticos y abióticos, tales como cambios en el suelo, la luz y la temperatura, relacionados con la apertura o el uso de senderos, que pueden causar cambios locales de la biodiversidad, además de afectar a los servicios de los ecosistemas generados por estas áreas. Sin embargo, el uso de herramientas de educación ambiental, la planificación de mantenimiento y revisión por parte del responsable de la Unidad de Conservación puede minimizar estos impactos y generar beneficios para la población local y visitantes en general, a través de prácticas de aprendizaje conservacionistas.

PALABRAS CLAVE: Ecoturismo. Educación Ambiental. Unidades de Conservación de Uso Público

\section{1- INTRODUÇÃO}

A maior conscientização dos valores ecológicos tem gerado uma maior busca por áreas naturais, principalmente através do uso de trilhas. As trilhas são historicamente essenciais ao homem na busca de alimento e água, e atualmente são cada vez mais procuradas para lazer, o que tem se intensificado devido a vida agitada nos centros urbanos (LADEIRA et al., 2007). Esse substancial aumento no volume de turistas que visitam áreas protegidas em busca de descanso e fuga da rotina vem causando grandes impactos a biodiversidade das Unidades de Conservação (UCs). De acordo com Vieira et al., 1999, a maior parte das áreas de uso público em UCs do Brasil, foram planejadas para atender um público menor e diferente do atual.

O uso de trilhas nas UCs favorece a maior aproximação do homem com a natureza possibilitando a realização de diversas atividades didáticas na área de Educação Ambiental (OLIVEIRA et al., 1999). Porém como discutido por Delgado (2000), apesar da utilização das áreas protegidas promover conscientização da 
população quanto a conservação dos ambientes naturais, a exploração dessas áreas como ferramenta para sensibilização da população pode causar danos as UCs devido a pressão que o uso das trilhas promove.

O uso irrestrito de recreação em áreas naturais tende a prejudicar as comunidades vegetais (COLE, 1978) e esse efeito tende a ser sentido a partir da abertura e da utilização das trilhas. Os impactos das trilhas sobre a vegetação podem ser diretos, por dano mecânico causado pela presença antrópica, ou indiretos, por mudanças causadas nas propriedades físicas e químicas do solo (RONCERO-SILES, 2003).

Há dois fatores principais de alteração do solo decorrentes da utilização de trilhas: compactação e erosão (COLE e LANDRES, 1995; HAMMITT e COLE, 1998). A compactação por pisoteio provoca remoção do horizonte orgânico do solo, o qual aumenta a absorção de água, diminui escoamento superficial, protege o horizonte mineral do solo, além de amenizar as forças compactadoras (COLE, 1993). A perda da porosidade parcial ou total, causada pela compactação dos solos (CURI 1993), está associada a redução do teor de matéria orgânica e ao efeito do impacto das gotas da chuva, diminuindo consequentemente a permeabilidade. Essa perda de permeabilidade diminui a capacidade de infiltração da água e, quando não há vegetação, aumenta o escoamento superficial da água das chuvas, o que causa a erosão (VASHCHENKO, 2006).

Roovers et al. (2004), alertaram para os efeitos da visitação intensiva sobre a compactação do solo e as consequentes alterações desses efeitos sobre a cobertura e a composição florística de uma determinada área, ressaltando ainda, que outros fatores bióticos e abióticos, como competição e fatores climáticos, possam ser fortemente alterados como resultado desse processo. Esses impactos podem afetar também a capacidade reprodutiva das espécies vegetais por interferir em processos como crescimento, germinação e estabelecimento de sementes (COLE 1978, ROOVERS et al., 2004). Assim, a intensidade do efeito causado pela trilha vai depender da vulnerabilidade da vegetação afetada, da frequência do uso da trilha e do regime de manutenção da mesma (COLE, 1978; ROOVERS et al., 2004). Os efeitos do pisoteio, decorrentes da visitação, incluem a redução da 
biomassa das plantas e a cobertura do solo; a diminuição na densidade de herbáceas, arbustos e plântulas; a substituição de espécies menos tolerantes por aquelas mais tolerantes ao impacto; e mudanças associadas ao solo que são frequentemente menos óbvias, como a perda de fauna e biomassa do solo, mas que com o tempo se tornam mais evidentes e mais preocupantes, (MAGRO, 1999).

A remoção da vegetação nativa para abertura da trilha também acarreta aumento na precipitação direta e na intensidade de luz, levando a alterações nas condições de drenagem, no ciclo de nutrientes e na dispersão por vetores (EISENLOHR, 2011). Além de danos a superfície, os turistas também afetam o complexo funcionamento da natureza, através da remoção por pisoteio ou coleta da vegetação nativa e de serrapilheira, que provém umidade e nutrientes ao solo, o que altera a dinâmica original do ambiente. Há também impactos mais sutis, como transporte de sementes de espécies exóticas, em sapatos, animais, rodas, entre outros; e algumas atividades como, barulho, oferta de alimentos, ou simplesmente a presença humana, podendo causar perturbações na fauna local.

Apesar de causar modificações notáveis nas comunidades florestais as trilhas no interior das matas são pouco estudadas no Brasil (EISENLOHR, 2011). Os impactos relacionados ao uso público das trilhas são variados. Segundo Cole (1978), possíveis sinais de estresse da vegetação podem ser mensurados diretamente por meio da comparação entre a vegetação imediatamente adjacente e a vegetação mais distante da trilha dentro da mesma formação vegetal. Outras modificações, como as causadas por competição intraespecíficas, são mais difíceis de detectar no ambiente, mas todas elas se constituem em importantes indicadores da saúde dos recursos naturais. De acordo com Eisenlohr (2011), dependendo da largura da trilha e da situação do fragmento em questão, efeitos próximos ou tão sérios quanto os efeitos de borda, podem ser notados devido a grande exposição a ação externa como, ventos mais fortes e maior luminosidade e temperatura, alterando a composição e estrutura da comunidade vegetal.

Assim, com o aumento nos últimos anos de visitações nas UCs, torna-se necessário buscar formas de lidar com os impactos causados pelos visitantes sem 
reduzir ou proibir o uso pela comunidade, visando novas formas de manejo que minimizem os impactos causados pela visitação.

\section{2- DESENVOLVIMENTO}

\section{1.- EDUCAÇÃO AMBIENTAL}

O uso de trilhas ecológicas é uma forma criativa e importante de educação ambiental tanto para as escolas, como para a população em geral. O uso de trilhas por professores é uma ótima maneira de aprendizagem, por possibilitar objetivos educacionais através da experiência prática, contextualizando o conhecimento passado nas aulas teóricas. Apesar disso, atualmente essa prática ainda é pouco usada pelas escolas.

As trilhas ecológicas podem se distinguir em interpretativas ou cênicas, de acordo com Lima (1998) "Trilhas de interpretação de caráter educativo consistem em instrumentos pedagógicos, podendo ser: (1) auto interpretativa ou auto guiada; (2) monitorada simples e guiada; (3) com monitoramento/guia associado a outras programações". Independentemente do tipo da trilha escolhida, ela desempenha importantes funções como, interagir os visitantes com o meio ambiente; criar maior compreensão e percepção dos recursos naturais; provocar mudanças de atitude, e atrair a população para ações de conservação.

Outra importante ferramenta de Educação Ambiental, utilizada pelas UCs, são os centros de visitação, que muitas vezes, possuem exposições, materiais impressos e vídeos que são repassados aos visitantes antes da utilização das trilhas. Essas informações têm sido cada vez mais criativas e interativas. As informações em forma de sinalização nas trilhas também transmitem importantes informações biológicas e mensagens conservacionistas aos visitantes.

As UCs, como um todo, têm se tornado cada vez mais favoráveis para práticas de Educação Ambiental. São nesses locais que as pessoas, de maioria residente no ambiente urbano, podem entrar em contato com a natureza (sendo as trilhas a principal atividade desenvolvida) e adquirirem informações sobre as interações que ocorrem no ambiente natural e as relações existentes entre 
sociedade e natureza (CAMPANHA, 2013). Através dos programas de educação ambiental também é possível conscientizar a população do entorno das UCs sobre a importância de se manter a área preservada, possibilitando a comunidade conhecer os seus gestores, projetos e ensinando o valor da conservação do ambiente em que vivem.

Desse modo, tanto para o ensino formal quanto para o não formal, as trilhas ecológicas constituem excelentes espaços para a prática de programas de Educação Ambiental. Essas práticas devem ir além de simplesmente ensinar o que os visitantes devem fazer durante a visita a UC, mas também propor mudanças no modo como as pessoas pensam e avaliam a sua relação com o meio ambiente (CAMPOS e FILLETO, 2011).

\section{2- INTERPRETAÇÃO AMBIENTAL}

A Interpretação Ambiental (IA) é considerada por alguns autores como recurso da educação ambiental, e tem ganhado espaço nos programas educacionais vinculados ao ecoturismo devido ao seu caráter educativo e recreativo (IKEMOTO, S. M. et al., 2009).

Tilde (1977) fundamentou a IA com o intuito de compreender como revelar significados, relações ou fenômenos naturais por intermédio de experiências práticas e meio interpretativos, ao invés da simples comunicação de dados e fatos. O mesmo autor, instituiu seis princípios para a elaboração do IA: (1) o objetivo principal não é o ensino, mas sim a sensibilização, estimulando o interesse e curiosidade do visitante; (2) o cotidiano deve ser correlacionado com a experiência vivida do visitante; (3) a interpretação deve apresentar os fatos na sua totalidade, evitando a fragmentação, assim como provocar a reflexão e o pensamento crítico do visitante; (4) a abordagem e o planejamento das atividades devem ser diferenciados de acordo com a faixa etária; (5) a IA é uma arte que se combina com várias outras, dessa forma se apropria de diversos materiais para o aprendizado, podendo ser científicos, históricos ou arquitetônicos; (6) a interpretação deve ser trabalhada de 
forma holística e integrada ressaltando as inter-relações e os elementos do ambiente.

A IA é considerada uma técnica didática desenvolvida por meio da apresentação de um ambiente com o auxílio de um intérprete, que irá esclarecer determinados fenômenos da natureza para um público alvo. A IA utiliza-se de uma linguagem adequada, de forma a promover a sensação de pertencimento à natureza, e assim gerar ao público, a consideração e respeito pela natureza. Desse modo, a IA possibilita a informação e a educação, e não apenas divertimento e distração (LIMA, 1998; PAGANI et al, 1999)

A IA pode estar presente em programas educacionais de diversas formas, sendo as "trilhas interpretativas" ou "trilhas de interpretação" um dos principais meios utilizados para a conscientização ambiental (Vasconcelos 1997; Takahashi 1997). Essas práticas têm como finalidade informar e sensibilizar para a conservação e/ou preservação do patrimônio cultural.

\section{3- COMUNIDADES LOCAIS}

A utilização do conhecimento da comunidade local tem auxiliado projetos de conservação em diversas linhas de pesquisas, como na quantificação da biodiversidade (MARTINS et al., 2001; NOVOTNY et al., 2002), monitoramento de impacto ambiental (BASSET et al., 2001), manejo sustentável, desenvolvimento de políticas para conservação (MOEF e UNEP, 2000), entre outras. De acordo com Sobrinho (2007), algumas experiências vêm mostrando que o sucesso das ações de conservação de um ecossistema, em um espaço protegido, depende da participação da população que habita o entorno dessas áreas e da forma com que essas pessoas se relacionam com o ambiente que as cercam.

Através de um estudo de caso em várias regiões do Brasil, Soares et al., (2002), encontrou que a atuação das populações locais do entorno de UC's, tem sido bastante positiva. De acordo com esses autores a participação da população local pode ser útil na manutenção e administração das UCs, inclusive auxiliando no plano de manejo, no desenvolvimento de projetos de educação ambiental, na 
participação da tomada de decisões, na manutenção dessas áreas e na construção de uma economia ecológica. Como discutido por Sobrinho (2007), devido ao conhecimento das matas do entorno das UCs, as populações vizinhas podem ser consideradas especialistas na flora local, reconhecendo diversas espécies e suas utilizações.

\section{4- TAXA DE ENTRADA}

Para Pires et al., (2004), os efeitos das atividades humanas nos ecossistemas são os principais responsáveis para que ele seja considerado sustentável ou não. A integridade ecológica dos ecossistemas naturais implica, necessariamente, na manutenção de suas áreas naturais com condições satisfatórias de qualidade ambiental, de forma a garantir a continuidade dos processos ecológicos ao longo do tempo (PIRES et al., 2004).

Lima 2003, fez um levantamento dos recursos financeiros de seis UCs em Minas Gerais, e conclui que $77 \%$ das UCs não recebem recursos suficientes para manter seu status, e buscar maior efetividade no manejo de suas áreas. O mesmo autor concluiu que muitas vezes a falta de recursos decorre da falta de vontade política dos dirigentes e está diretamente relacionada as UCs que não existem efetivamente, os chamados "parques de papel".

Em um estudo mais recente em 30 UCs no corredor central da Mata Atlântica no estado da Bahia, Schiavetti et al., (2012) encontraram que a situação financeira das UCs era insustentável, com cerca de $30 \%$ sem nenhuma receita, e as que possuíam, o valor frequente era insuficiente para provimento de atividades como manutenção e aquisição de equipamentos de infraestrutura e escritório, fiscalização e projetos. Desse modo, a cobrança de taxas pode funcionar como um importante mecanismo para complementar os recursos necessários para a manutenção destas áreas. As taxas podem ser cobradas de diferentes formas como, ingressos, realização de atividades recreativas, camping, taxas de concessão de serviços, venda de alimentos e mercadorias (EAGLES et al., 2002). 


\section{5- ECOTURISMO E ESPORTES RADICAIS}

O ecoturismo, como outras formas de turismo, pode ser uma fonte de renda bastante instável. Muitos fatores externos podem influenciar a demanda turística, alguns desses fatores estão completamente fora do controle das destinações turísticas, no entanto, afetam os níveis de visitação. Por exemplo, falta de segurança em uma região, ou desastres naturais, como tempestades, podem facilmente destruir a infraestrutura turística em determinada áreas e desse modo desencorajar os visitantes.

O uso de veículos (bicicletas, motocicletas, automóveis) provocam várias alterações no solo como, compactação, aumento da densidade e resistência, mudanças na estrutura e na sua estabilidade, diminuição no acumulo de serrapilheira e conteúdo de húmus, e redução nas taxas de infiltração, o que aumenta o escoamento hídrico superficial causando a erosão (GUALTIERI-PINTO et al., 2008). Essas alterações variam de acordo com os tipos de usos que a trilha é submetida. Segundo Marion e Olive (2006), trilhas cujo uso está concentrado em atividades de caminhada e ciclismo geralmente apresentam pouca degradação relacionada a atividade erosiva, quando comparada a trilhas onde há presença constante de cavalos e veículos off-road. De acordo com Gualtieri-Pinto et al., (2008), outra variável importante são os tipos de solos, por exemplo, os solos arenosos e pouco coesos podem ter maior propensão a perda de sedimentos, ocasionando o aprofundamento do leito da trilha, dificultando assim, o trânsito de usuários. Essas modificações, podem induzir os usuários a procurar as áreas adjacentes a trilha principal, originando assim, trilhas secundárias que podem resultar, no futuro, em exposição do solo no leito da trilha e desenvolvimento de novos focos erosivos.

Como nos dias atuais a atividade ecoturística vem sendo muito difundida, torna-se necessário um conhecimento mais específico das condições físicas das trilhas, sendo o monitoramento da atividade erosiva no leito destas, uma importante variável para que se conheça seu estado de conservação e de segurança para as pessoas que as utilizam. 


\section{3 - CONCLUSÃO}

A utilização pública de trilhas em UCs é uma combinação de oportunidades e ameaças. As trilhas podem ser consideradas um paradoxo à conservação e restauração dos ambientes naturais (EISENLOHR et al., 2013), representando uma ferramenta a favor da conservação e restauração por permitirem a conscientização ambiental, e ao mesmo tempo servindo também como fonte de distúrbio a esses ambientes (EISENLOHR et al., 2009, 2011). Portanto, para amenizar os impactos provocados pelos visitantes é necessário que haja um planejamento adequado, visando avaliar a suscetibilidade das comunidades (animal e vegetal), as condições físicas das trilhas e para qual fim está sendo utilizada. Desse modo, a continuidade das atividades de manutenção e fiscalização das trilhas são essenciais para a manutenção das UCs, gerando benefícios para os visitantes e garantindo seu principal objetivo de conservação da biodiversidade. Além disso, conhecer as características dos visitantes como, idade, sexo, escolaridade, objetivo da visita, tempo de permanência e percepção ambiental, pode ajudar os gestores das UCs a compreender as causas e potenciais soluções dos impactos ecológicos e recreativos.

\section{REFERÊNCIAS}

Alves, Ligia Martins. Trilha Interpretativa da Embrapa ("trilhas da matinha") Dourados, MS: Contexto para Educação Ambiental, Campo Grande-MS. Dissertação (Mestrado) -Faculdade Federal do Mato Grosso do Sul. Campo Grande, 2013. 101 p.

ANDRADE, Waldir Joel. Implantação e manejo de trilhas. In: S. Mitraud (org.). Manual de ecoturismo de base comunitária: ferramentas para um planejamento responsável. WWF-Brasil, Brasília, p.247-259, 2003

BASSET, Yves et al. Monitoring species loss after forest disturbance. Short-term effects of canopy openness on insect herbivores in a rain forest in Guyana. Journal of Applied Ecology. v.38, p.1045-1058, 2001.

CAMPANHA, Leticia Francisca Pezzi. Trilhas Ecológicas como recurso de Educação Ambiental. Universidade Estadual de Londrina. Paraná, 2013. 9 p. 
CAMPOS, Renata Ferreira; FILLETO, Ferdinando. Análise do perfil, da percepção ambiental e da qualidade da experiência dos visitantes da Serra do Cipó (MG). Revista Brasileira de Ecoturismo v.4, p.69-94, 2011.

CARVALHO, Joema; BÓÇON, Roberto. Planejamento do traçado de uma trilha interpretativa através da caracterização florística. Revista Floresta v.34, p.23-32, 2004.

COLE, David. Estimating the susceptibility of wildland vegetation to trailside alteration. The Journal of Applied Ecology v.15, p.281-286, 1978.

COLE, David N. Minimizing conflict between recreation and nature. In: SMITH Daniel; HELLMUND, Paul Cawood. (Eds). Ecology of greenways: design and function of linear conservation areas. Minneapolis: University of Minnesota Press. p.105-122, 1993.

COLE, David; LANDRES Peter. Indirect effects of recreation on wildlife. In: KNIGHT, Richard.; GUTZWILLER, Kevin (Eds). Wildlife and recreationists: coexistence through management and research. Washington: Island Press. p.183-202, 1995.

CURI, Nilton. Vocabulário de Ciência do Solo. Campinas: Sociedade Brasileira de Ciência do solo. Campinas, 1993. 89 p.

DELGADO, Jesus. A interpretação ambiental como instrumento para o ecoturismo. In: C. Serrano. A educação pelas pedras. Chronos, São Paulo, p.155-169, 2000.

EAGLES, Paul; MC COOL, Stephen; HAYNES, Cristopher. Turismo sostenible en áreas protegidas: directrices de planificación y gestión. Madrid: PNUMA-OMT-UICN, 2002. 218 p.

EISENLOHR, Pedro Vasconcellos.; MELO, Maria Margarida Rocha Fiuzza.; SILVA, Adriano Valentin. Trilhas afetam comunidades arbóreas Florestais? Dois levantamentos na Floresta Atlântica do sudeste brasileiro. Hoehnea v.36, p. 293-302, 2009.

EISENLOHR, Pedro Vasconcelos et al. Floristic variations in a woody plant community along a trail in a Semideciduous Seasonal Forest, Viçosa, Minas Gerais State, Brazil. Hoehnea v.38, p. 61-71, 2011.

EISENLOHR, Pedro Vasconcelos et al. Trilhas e seu papel ecológico: o que temos aprendido e quais as perspectivas para a restauração de ecossistemas? Hoehnea v.40, p.3, 2013.

HAMMITT, Willian; COLE, David. Wildland recreation: ecology and management. 2 ed. New York: John Wiley \& Sons, 1998. $361 \mathrm{p}$.

IKEMOTO, Silvia Marie; MORAES, Moemy Gomes; COSTA Vivian Castilho. Avaliação do Potencial Interpretativo da Trilha do Jequitibá, Parque Estadual dos Três Picos. Sociedade \& Natureza v.21, p.271-287, 2009.

LADEIRA, Alecia Silva et al. O perfil dos visitantes do Parque Estadual do Ibitipoca (PElb), Lima Duarte, MG. Revista Árvore v.31,p.1091-1098, 2007.

LIMA, Gumercindo Souza. Criação, implantação e manejo de Unidades de Conservação no Brasil: Estudo de caso em Minas Gerais. Tese (Doutorado) Universidade Federal de Viçosa, Viçosa, 2003. 
LIMA, Solange Guimarães. Trilhas Interpretativas: A aventura de conhecer a Paisagem. Cadernos Paisagem, v.3, 1998.

MAGRO, Teresa Cristina. Impactos do uso público em uma trilha no planalto do Parque Nacional do Itatiaia. 1999. Tese (Doutorado) - Escola de Engenharia de São Carlos, Universidade de São Paulo. São Carlos, 1999.

MARION, Jeffrey; OLIVE, Nate. Assessing and understanding trail degradation: results from Big South Fork National River and recreational area. USGS Patuxent Wildlife Research Center, Virginia. 2006.

MARTINS, Gary et al. Projek Etnobotani Kinabalu: The Making of a Dusun Ethnoflora (Sabah, Malaysia). People and Plants Working Paper, UNESCO. 2001.

MOEF; UNEP. National Biodiversity Strategy and Action Plan. UNEP. 2000.

NOVOTNY; Vojtech et al. Low host specificity of herbivorous insects in a tropical forest. Nature v.416, p.841-844, 2002.

OLIVEIRA, Renata Teixeira; BLOOMFIELD, Vanessa Kelva; MAGALHÃES, Luis Mauro Sampaio. Trilhas auto-guiadas: proposta de implantação e interpretação na Floresta Nacional Mário Xavier Sandra Regina da Costa. Floresta e Ambiente v.6, p.138-143, 1999.

PAGANI, Maria Inez et al. As trilhas interpretativas da natureza e o ecoturismo. In: Lemos, Amália Inês. (Org.). Turismo: Impactos Sócio Ambientais. São Paulo: Hucitec, p.151-163, 1996.

PIRES, José Salatiel et al. Gestão biorregional. Uma abordagem conceitual para o manejo de paisagens. In: Santos et al J.E. (orgs.). Faces da polissemia da paisagem: Ecologia, planejamento e percepção. RIMA, São Carlos, p.23-34, 2004.

RONCERO-SILES, Maria Francisca. Efeitos do pisoteio humano experimental sobre a vegetação em fragmentos de Floresta Pluvial Tropical Atlântica, São Paulo, Brasil. 2009. Tese (Doutorado) Universidade de São Paulo. São Paulo, 2009.

ROOVERS, Pieter; BAETEN, Sara; HERMY, Martin. Plant species variation across path ecotones in a variety of commom vegetation types. Plant Ecology v.170, p.107-119, 2004.

SCHIAVETTI, Alexandre et al. Implementação das unidades de conservação do corredor central da mata atlântica no estado da bahia: desafios e limites. Revista Árvore, v. 4, p.611-623, 2012.

SOARES, Maria Clara Couto; BENSUNAM, Nurit; FERREIRA NETO, Paulo Sergio. Entorno de Unidades de Conservação: estudos de experiências com UC's de Proteção integral. Rio de Janeiro: FUNBIO, 112 P (Série estudos FUNBIO). 2002.

SOBRINHO, Felipe Araújo Pinto. Conhecimento etnobotânico de mateiros residentes no entorno de Unidades de Conservação no estado do Rio de Janeiro. 2007. Dissertação (Mestrado). Jardim Botânico do Rio de Janeiro. Rio de Janeiro, 2007.

TAKAHASHI, Leide Yassuco. Limite Aceitável de Câmbio (LAC): Manejando e Monitorando Visitantes. I Congresso Brasileiro de Unidades de Conservação. Anais. Curitiba, p.445-464, 1997.

TILDEN, Freeman. Interpreting our heritage. North Carolina: The University of North Carolina Press, 1977. $120 \mathrm{p}$. 
VASCONCELLOS, Jane. Trilhas interpretativas: aliando educação e recreação. In: Congresso Brasileiro de Unidades de Conservação, 1, 1997, Curitiba. Anais... Curitiba: IAP, UNILIVRE, REDE PRÓ-UC, v1. p.465-477, 1997.

VASHCHENKO, Yury. Caracterização da trilha e o impacto do montanhismo nos Picos Camapuã e Tucum - Campina Grande do Sul - PR. Dissertação (Mestrado) - Faculdade de Ciências Agrárias, Universidade Federal do Paraná. Curitiba,106pp, 2006.

VIEIRA, Valeria Maradei Freixêdas; PASSOLD, Ana Julia; MAGRO, Teresa Cristina. Impactos do uso público: Um guia de campo para utilização do método VIM. In: Congresso Brasileiro de Unidades de Conservação, 2. Anais... Campo Grande: Rede Nacional Pró-Unidades de Conservação: Fundação O Boticário de Proteção à Natureza, v3, 2000. 